Notre Dame Journal of Formal Logic

Volume XXI, Number 1, January 1980

NDJFAM

\title{
GENERALIZED EQUIVALENCE AND THE FOUNDATIONS OF QUASIGROUPS
}

\author{
T. A. McKEE
}

1 Introduction We define the generalized equivalence of a finite set $\&=\left\{E_{1}, \ldots, E_{n}\right\}$ of statements, denoted $[\&]$ or $\left[E_{1}, \ldots, E_{n}\right]$, to be the conjunction for all $i \leqslant n$ of the implications $\wedge\left(\&-\left\{E_{i}\right\}\right) \rightarrow E_{i}$, where $\wedge \mathcal{F}$ denotes the conjunction of the elements of set $\mathcal{J}$. Thus $\left[E_{1}\right]$ is $E_{1},\left[E_{1}, E_{2}\right]$ is $\left(E_{1} \rightarrow E_{2}\right) \&\left(E_{2} \rightarrow E_{1}\right)$, and $\left[E_{1}, E_{2}, E_{3}\right]$ is $\left(E_{1} \& E_{2} \rightarrow E_{3}\right) \&\left(E_{2} \& E_{3} \rightarrow\right.$ $\left.E_{1}\right) \&\left(E_{1} \& E_{3} \rightarrow E_{2}\right)$. The elements of a set of statements are generalized equivalent exactly when their generalized equivalence is true; that is, whenever each statement is implied by the conjunction of all the others. (In practice it is sometimes useful to note that the elements of \& are generalized equivalent exactly when the conjunction of each proper subset $\delta^{\prime}$ of $\&$ implies the generalized equivalence of the elements of $\&-\delta^{\prime}$.) For example, in a finite dimensional vector space, the statements asserting independence of a subset, spanning the space, and having cardinality equal to the dimension are generalized equivalent.

The importance of generalized equivalence in quasigroups is suggested by the definition of a quasigroup as a set with a binary operation (denoted by juxtaposition) such that, in the equality $a b=c$, each of the three elements is uniquely determined by the other two; see [1] or [3]. This is just the definition of closure under a binary operation together with what could be called its two converses. As a simple example of a quasigroup, consider the set of points of the Euclidean plane with the binary operation on two points giving the midpoint of the segment joining them.

Quasigroups are typically studied under "constraints" such as $a(b c)=$ $(a b) c$. (In fact, being an associative quasigroup is equivalent to being a group.) Frequently the constraint is stated in terms of implications between equalities, and very often such implications can be automatically "strengthened"' to equivalences. For instance, the constraint

$$
a b=c d \rightarrow a(b x)=c(d x)
$$

can be shown equivalent to 


$$
a b=c d \leftrightarrow a(b x)=c(d x)
$$

And the more complex constraint

$$
a b=a^{\prime} b^{\prime} \& a c=a^{\prime} c^{\prime} \& b d=b^{\prime} d^{\prime} \rightarrow c d=c^{\prime} d^{\prime}
$$

is equivalent to the generalized equivalence of the four equalities $a b=a^{\prime} b^{\prime}$, $a c=a^{\prime} c^{\prime}, b d=b^{\prime} d^{\prime}$, and $c d=c^{\prime} d^{\prime}$. That this is so can be shown by a quasigroup argument or as an instance of our Corollary in Section 3. (These and all other constraints which we mention are discussed in [3].)

This tendency of implications to be interchangeable with (generalized) equivalences is rather unexpected. Our intent is to view this phenomenon in terms of the relationship between quantifiers and generalized equivalence. (This relationship will also be used in a different way in the subsequent article [2] concerning the profusion of generalized equivalences in configuration theorems of projective geometry.)

2 Productive and configurational sets of equations An equality is a pair of expressions, separated by $=$, each built from a binary operation (and parentheses); for instance, $a(b(c d))=a$. An atomic equality is an equality involving only one operation; for instance, $a b=c$. It is often useful to "parse" an equality into a set of atomic equalities by the introduction of new variables (see Section 6 of [3]). For example, $a b=c d$ can be parsed into $\{a b=u, c d=u\}$ and $(x a)(x b)=(y c)(y d)$ into $\{x a=p, x b=q, p q=v$, $y c=s, y d=t, s t=v\}$. We next introduce the notion of "productive" which captures the essence of sets of atomic equalities obtained in this way.

We shall call a set $\&$ of two or more atomic equalities a productive set (with producing variables $a, b, c, \ldots$ and produced variables $u, v, w, \ldots$ ) whenever it satisfies all the following:

P1 A variable does not occur more than once within a single element of $\&$, nor do the same two variables occur in two different elements.

P2 Each produced variable occurring in \& occurs exactly twice.

P3 Every two elements of $\&$ are linked by a sequence of elements of $\&$ with each pair of consecutive terms of the sequence sharing a common produced variable.

P4 The cardinality of $\&$ is one more than the number of produced variables occurring in $\&$.

It is convienent to consider a graph with the atomic equalities serving as vertices and with two vertices joined by an edge precisely when they share a common produced variable. Then a productive set corresponds to a tree graph, with the produced variables serving as edges. The following are examples of productive sets: $\delta_{1}=\{a b=u, c d=v, u v=w, a c=x$, $b d=y, x y=w\}$ and $\&_{2}=\{a b=x, a c=y, x y=z, a w=z, b c=w\}$.

Let $\forall \Phi$ and $\exists \Phi$ be, respectively, the universal and existential closures of $\Phi$, with the quantification ranging over all the variables which have not been interpreted as fixed elements of a quasigroup. For instance, if $a, b$, 
and $c$ have been interpreted (that is, assigned values) as elements of a quasigroup 2 , then $\forall\left[\mathscr{\&}_{2}\right]$, where $\&_{2}$ is as above, is

$$
\forall x \forall y \forall z \forall w[a b=x, a c=y, x y=z, a w=z, b c=w],
$$

where the quantifiers range over the elements of 2 . Note that if equality $E$ is parsed into the set \& of atomic equalities as explained above, then $\&$ is productive with the new variables as produced variables; also $E$, with its original variables interpreted as elements of 2 , will be equivalent to each of $\forall[\&]$ and $\exists \wedge \&$.

Lemma Suppose \& is a productive set (of atomic equalities) whose producing variables have been interpreted in a quasigroup 2 . Then

L1 For each maximal proper subset \&* of \&, the produced variables of \& can be assigned uniquely in 2 so as to satisfy $\wedge \& *$.

L2 2 satisfies $\forall[\&]$ if and only if 2 satisfies $\exists \wedge \&$.

To prove L1, suppose \& is productive with its producing variables interpreted in 2 . Since each atomic equality contains three different variables, the number of different occurrences of (possibly repeated) producing variables in $\&$ is three times the cardinality of $\&$, minus twice the number of produced variables, and so by $\mathrm{P} 4$ equals two greater than the cardinality of $\&$. Since by P3 none of the equalities can involve three producing variables, at least two equalities in \& must each involve exactly two producing variables. Then any maximal proper subset $\&^{*}$ of $\&$ will contain some equality $E$ which involves exactly two producing and so exactly one produced variable. Since 2 is a quasigroup, this variable can be assigned a unique value in 2 so as to satisfy $E$. If $\&^{*}$ contains more than one equality, then $\&-\{E\}$ will be a smaller productive set, with $E$ 's produced variable now viewed as a producing variable. Continuing in this fashion will eventually assign unique values to all the produced variables of $\&$ so as to satisfy $\wedge \& *$.

To prove the "only if" direction of L2, assume $\forall[\&]$ and let $\& *$ be any maximal proper subset of $\&$. By L1 we know there is an assignment of $\&$ 's produced variables which will satisfy $\wedge_{\&^{*}}$ and so, by the assumption $\forall[\&]$, it will satisfy $\wedge \&$, thereby showing $\exists \wedge \&$.

Conversely, assume elements $q_{1}, q_{2}, \ldots$ from 2 satisfy all of $\&$ and that elements $r_{1}, r_{2}, \ldots$ satisfy all of a maximal proper subset $\&^{*}$ of $\&$. By the uniqueness of L1, each $q_{i}=r_{i}$ and so $r_{1}, r_{2}, \ldots$ satisfy all of $\&$, thereby showing $\forall\left[\&^{\prime}\right]$.

(In connection with $\mathrm{L} 2$, it is interesting to note that an atomic equality can itself be viewed as a generalized equivalence. In the notation of [3], $a b=c$ is equivalent to each of $(\forall t \in G)[\&]$ and $(\exists t \in G) \wedge \&$, where \& $=$ $\left\{a=\pi_{1}(t), b=\pi_{2}(t), c=\pi_{3}(t)\right\}$.)

Theorem Suppose $\&_{1}, \ldots, \&_{k}$ are disjoint productive sets such that each produced variable occurring in $\delta_{1} \cup \ldots \cup \&_{k}$ occurs exactly twice. Then for each quasigroup 2 , the following are equivalent: 
$\mathrm{T} 1$ For each 2-interpretation of the producing variables of $\&_{1}, \ldots, \&_{k}$, $\left[\forall\left[\&_{1}\right], \ldots, \forall\left[\&_{k}\right]\right]$.

T2 For each 2-interpretation of the producing variables of $\&_{1}, \ldots, \&_{k}$, $\forall\left[\&_{1} \cup \ldots \cup \&_{k}\right]$.

To prove that $\mathrm{T} 1$ implies $\mathrm{T} 2$, assume $\mathrm{T} 1$ and that the producing variables are interpreted in 2 and that (for $i \leqslant k$ ) elements $q_{i 1}, q_{i 2}, \ldots$ satisfy all but (possibly) one equality of $\delta_{1} \cup \ldots \cup \&_{k-1}$; for conciseness, say they satisfy all of $\delta_{1} \cup \ldots \cup \&_{k-1} \cup \&_{l k}^{*}$ where $\delta_{1}^{*}$ is a maximal proper subset of $\delta_{k}$. So for $i<k$ we have $\exists \wedge \&_{i}$ and so by L2 we have $\forall\left[\&_{i}\right]$ for $i<k$, and so by $\mathrm{T} 1$ we have $\forall\left[\delta_{k}\right]$. Since $q_{k 1}, q_{k 2}, \ldots$ satisfy $\wedge \&_{k}^{*}, \forall\left[\&_{k}\right]$ shows they also satisfy $\wedge \&_{k}$, thereby showing T2.

Conversely, assume $\mathrm{T} 2$, that the producing variables are interpreted in 2, $\forall\left[\&_{i}\right]$ for $i<k$, and that $q_{k 1}, q_{k 2}, \ldots$ satisfy $\wedge_{\delta_{k}^{*}}^{*}$ where $\delta_{k}^{*}$ is a maximal proper subset of $\delta_{k}$. Applying L2 for each $i<k$ gives $q_{i 1}, q_{i 2}, \ldots$ satisfying each $\Lambda_{i} \&_{i}$ for $i<k$. So all the elements $q_{i 1}, q_{i 2}, \ldots(i \leqslant k)$ satisfy a maximal proper subset of $\delta_{1} \cup \ldots \cup \delta_{k}$ and so, by T2, all of it. In particular, $q_{k 1}, q_{k 2}, \ldots$ satisfy $\wedge \wedge_{k}$, thereby showing $\forall\left[\& \&_{k}\right]$ and so $\mathrm{T} 1$.

We shall call a set \& of atomic equalities a configurational set whenever it satisfies all the following:

C1 A variable does not occur more than once within a single element of \&; nor do the same two variables occur in two different elements

C2 Each variable occurring in \& occurs exactly twice

C3 Every two elements of $\&$ are linked by a sequence of elements of $\&$ with each pair of consecutive terms of the sequence sharing a common variable.

A configurational set may be viewed as a graph with the equalities serving as vertices and with two vertices joined by an edge precisely when they share a common variable. The definition of configurational ensures that the graph will be connected and trivalent. The edges of any spanning tree can be taken as produced variables, and the set can then be viewed as productive. The set $\delta_{1}$ given above is an example of a configurational set, while $\&_{2}$ is not.

We shall call a set of (not necessarily atomic) equalities a configurational set if the union of the sets into which its elements are parsed is a configurational set of atomic equalities. Thus, for instance, $\{a b=c d$, $(x a)(x b)=(y c)(y d)\}$ is a configurational set.

3 Applications to quasigroups We are now in the position to discuss the phenomenon mentioned in the introduction.

Corollary Suppose $\left\{E_{1}, \ldots, E_{k+1}\right\}$ is a configurational set of equalities. Then $\forall\left(E_{1} \& \ldots \& E_{k} \rightarrow E_{k+1}\right)$ is equivalent to $\forall\left[E_{1}, \ldots, E_{k+1}\right]$.

To prove the Corollary, note that we need only show that the implication implies the generalized equivalence. We first consider the special 
case in which each $E_{i}$ is an atomic equality. Put $\&=\left\{E_{1}, \ldots, E_{k+1}\right\}$ and use any spanning tree of \&'s associated graph so as to view \& as productive. Assume the producing variables are arbitrarily assigned values in a quasigroup 2 satisfying $\forall\left(\wedge_{\&^{*}} \rightarrow E_{k+1}\right)$, where $\&^{*}=\left\{E_{1}, \ldots, E_{k}\right\}$. Then this assumption and Lemma L1 show that 2 satisfies $\exists \wedge \&$ and so $\forall[\&]$ by $\mathrm{L} 2$.

To prove the general case, let $\&_{i}$ be the productive set obtained by parsing $E_{i}$. Then $\&=\&_{1} \cup \ldots . \cup \&_{k+1}$ is a configurational set of atomic equalities. Suppose a quasigroup 2 satisfies $\forall\left(E_{1} \& \ldots \& E_{k} \rightarrow E_{k+1}\right)$ so that, for any 2-assignment of the variables, if all the elements of $\&_{1} \cup \ldots \cup \&_{k}$ and of a maximal proper subset of $\&_{k+1}$ hold in 2 , then so will all of $\&$. Then by the previously proved special case, $\forall[\&]$. So the Theorem implies that $\left[\forall\left[\&_{1}\right], \ldots, \forall\left[\&_{k+1}\right]\right]$ for each 2 -interpretation, and so $\forall\left[E_{1}, \ldots, E_{k+1}\right]$.

The special (atomic) case of the Corollary compares with Stein's observation (a) on page 249 of [3]. His observation (b) then matches the equicardinality of the spanning trees in the graph associated with the configurational set.

As another application of the Theorem, consider the constraint $\forall[(a b) c=a d,(e b) c=e d]$, which is the third constraint in (18.1) of [3]. As in the proof of the Corollary, this can be rewritten

$$
\forall[a b=x, x c=u, a d=u, e b=y, y c=v, e d=v] .
$$

Since this configurational set of six atomic equalities can be partitioned into the two productive sets $\&_{1}=\{a b=x, x c=u, a d=u\}$ and $\&_{2}=\{e b=y$, $y c=v, e d=v\}$ with the produced variables $x, u$ and $y, v$, respectively, the Theorem shows the original constraint equivalent to $\forall\left[\forall x, u\left[\&_{1}\right], \forall y, v\left[\&_{2}\right]\right]$. This corresponds to the (13) conjugate constraint of the original constraint in [3]. Similarly, the set can be partitioned into three productive sets, resulting in the constraint

$$
\forall[\forall b[a b=x, e b=y], \forall c[x c=u, y c=v], \forall d[a d=u, e d=v]],
$$

corresponding to the (23) conjugate of the original in [3].

It should be kept in mind that a constraint may be written in very different ways as universally-quantified generalized equivalences of atomic equalities. For instance the constraint

$$
\forall(a b=c d \rightarrow(x a)(x b)=(y c)(y d))
$$

can be written as

$$
\forall[a b=u, c d=u, x a=p, x b=q, p q=r, y c=s, y d=t, s t=r] .
$$

But by Corollary 7.6 of [3] it may also be written as

$$
\forall[a b=u, a c=v, u v=w, x y=w, d b=x, d c=y] .
$$

Because of the role of generalized equivalence in quasigroup theory, other possible uses in the context of [3] are suggested. For instance, a constraint is commonly called invariant if it is equivalent to each of its 
conjugates. What about the notion of the generalized equivalence of the six conjugates of the constraint-that is, "generalized invariance"? Or what can be said of the quasigroups which satisfy the constraint

$$
[a b=c, b a=c, c b=a, a c=b, b c=a, c a=b] ;
$$

that is, of "generalized totally symmetric" quasigroups?

\section{REFERENCES}

[1] Bruck, R. H., A Survey of Binary Systems, Springer, Berln, 1958.

[2] McKee, T. A., "Generalized equivalence and the phraseology of configuration theorems," Notre Dame Journal of Formal Logic, vol. XXI (1980), pp. 141-147.

[3] Stein, S. K., "On the foundations of quasigroups," Transactions of the American Mathematical Society, vol. 85 (1957), pp. 228-256.

Wright State University

Dayton, Ohio 